\title{
Purification capability of broccoli to gaseous ethyl alcohol using an LED lighting system
}

\author{
T. Oyabu \\ Kanazawa Seiryo University, Japan
}

\begin{abstract}
Plants have various kinds of functions. One of them is a purifying function to gaseous contaminants including carbon dioxide. It is a noticeable function from the standpoint of protection of the global environment. Plants can purify not only carbon dioxide but also nitrogen oxide and sulfur oxide, and the chemicals are used as a source of energy after having been broken down in the plants. In addition, plants put out a bioelectric potential which changes depending on the environment and growing conditions. In this study, the relationship between the potential and purification capability of a plant is examined using an LED lighting system. Broccoli is adopted as a subjective plant and the experiment is carried out with five kinds of LED light frequencies including darkness. The summation value is adopted as the potential value, namely the total amount of the bioelectric potential for a minute $\left(v_{m 1}\right)$. As a result, the capability increases and the potential also becomes greater in blue light. A positive correlation is recognized in the relationship between the potential and the capability. A specific gaseous chemical could be purified in a particular light-frequency by applying the results of this study.
\end{abstract}

Keywords: plant bioelectric potential, air pollution, purification capability.

\section{Introduction}

Plants (vegetation) have various kinds of functions which contribute to the welfare of humans. The origins of terrestrial plants go back hundreds of millions of years. Meanwhile, humans, as homo sapiens, have only been around for the last 200 thousand years [1]. Terrestrial plants have been in existence for a long period of time, and they contribute to the welfare of humans, for example, with food, fuel, medicine and air purification by photosynthesis. Recently, global warming has become a major issue and the purifying function of plants for the 
warming chemicals is in the limelight. If the photosynthesis mechanism of plants can be simulated and the equipment can purify not only air pollutants but also carbon dioxide in the same way as plants do, the mechanism is very effective. Humans enjoy the benefit of air purification and the healing effects due to plants [2]. Humans have been living a long time with plants, so we have a healing effect on plants.

Recently, vegetable factories have been set up in Japan with the purpose of improving the food self-sufficiency ratio [3-9]. The system has a big appeal when vegetables are produced in the factory. If carbon dioxide in the atmosphere can be compressed and used in the factory, it has great effect. Carbon dioxide is essential for vegetable growing and it is apparent that the irradiating light frequency plays a key role in the growth and purifying function of vegetables [10]. The concentration of carbon dioxide also plays a significant role for plant growth. In this study, it is shown that there is a correlation between the irradiating light frequency and the plants' bioelectric potential. It is contemplated that the potential derives from an ion stream caused by the vital activity of the plants, similar to an electrocardiogram [11]. The potential varies with the environmental condition and physiological state of the plants. There is a possibility to control the plant growth using the results in a vegetable factory. Bioelectric potential is the only information transmitted by plants. Practical farmers can evaluate instantly the growth grade, the extent of disease and harvest time of agricultural products by visual observation. They have the knowledge on the growing vegetable based on experimental grounds. If we can evaluate the described items (plant conditions) utilizing the potential, even a complete amateur can control plant growth and the harvesting.

In this investigation, the bioelectric potential for the light frequency is examined to apply the results to production management of vegetables in years to come [12]. And the relation between the potential and purification capability is also examined [13]. In this paper, the relation is studied specifically when the frequency of irradiation light is changed. Broccoli is adopted as a subjective plant which is a familiar food and ethyl alcohol is used as an air pollutant.

\section{Experimental}

The experiment on the plant-bioelectric potential was carried out in an acrylic chamber (575x510x1000 mm, about 300 liter). The external light (e.g. sunlight, fluorescent lights) was cut off in the experiment. The system is shown in Fig. 1 [10]. Broccoli (species: stick senor) was adopted as the subjective vegetable. It is a variant of cabbage and a deep yellow vegetable. The potential for cabbage was measured and reported [10]. A photograph of the subjective plant is shown in Fig. 2. Broccoli has hard leaves and the electrodes can be easily assembled on the leaf. The electrodes are installed to the neighbouring leaves and the contact area is $0.3 \mathrm{~cm}^{2}$. The soil of pot is ground.

Plants absorb and break down atmospheric polluting chemicals and a portion of them is received into the body tissue as nourishment [14]. Ethyl alcohol $\left(\mathrm{C}_{2} \mathrm{H}_{5} \mathrm{OH}\right.$, acceptable atmospheric concentration limit is $\left.1,000 \mathrm{ppm}\right)$ is adopted 
as a polluting chemical. The substance is used as a rubbing alcohol and the gas sensor has a high sensitivity to it. Carbon dioxide and nitrogen oxide are generally used. The potential is several millivolts which is amplified 100 -fold using a difference amplifier. Therefore, it oscillates centering on zero level and high-frequency noise level is removed by a low pass filter $(10 \mathrm{~Hz})$. The signal is input into a mobile computer through an A-D converter and data logger. It is difficult to derive the feature by analyzing the signal directly. The new value $\left(v_{m 1}\right)$ is derived, namely the summation of the signal for a minute. The sampling interval is 0.1 second and the $v_{m 1}$ is analyzed to derive the quality. The following sensors are installed to measure the environmental factors in the experimental chamber, namely tin oxide gas sensor (Figaro, TGS \#800), temperature and humidity sensors with atmospheric pressure meter (T\&D, TR-73U) and illuminometer (T\&D, PHR-51). The sampling interval for the sensors is every minute. Four kinds of LED panels are adopted as an irradiation light source, namely blue (475 nm), green (525 nm), red (660 nm) and white lights. And the experiment is also carried out in darkness (black). The panel is set up at the top of the chamber and the height is $90 \mathrm{~cm}$ from the bottom. Temperature, humidity, atmospheric pressure and concentration of ethyl alcohol in the chamber are measured at the bottom position [10].

The purifying capability of the broccoli under each light frequency is derived using the gas sensor output $\left(v_{s}\right)$. The gas sensor output without ethyl alcohol is called the "offset level" ( $\left.v_{\text {off }}\right)$, and the difference between the maximum output $\left(v_{s}\right.$-max) when the chemical is injected into the chamber and the offset level is named as "h". The $v_{s}$-max is derived in a matter of seconds in the case of ethyl alcohol. The half-value width of $\mathrm{h}\left(=v_{\mathrm{s}-\max }-v_{\text {off }}\right)$ is called as $\mathrm{t}_{\mathrm{w}}$ and the purification capability $\left(P_{a}\right)$ is introduced using equation (1) [15]. The general description is indicated in Fig. 3. In the figure, part (a) shows the condition without ethyl alcohol and part (b) is after the injection of the chemical, so the level becomes the maximum value at once and decreases gradually. The new potential $\left(v_{m 1}\right)$ has an increasing trend when there is a chemical. The average $\left(v_{m 1-\text { lave }}\right)$ and histogram of $v_{m 1}$ are utilized for the analysis.

$$
\mathrm{P}_{\mathrm{a}}=\mathrm{h} / \mathrm{t}_{\mathrm{w}} \times 100
$$

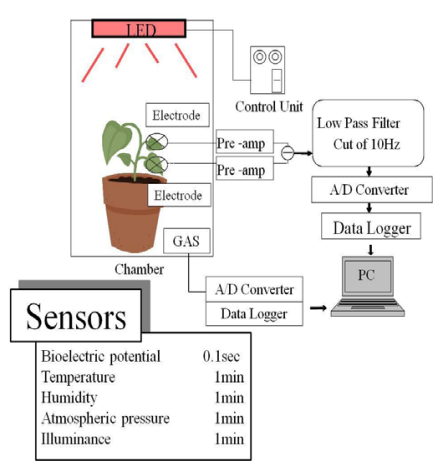

Figure 1: $\quad$ Schematic diagram of the experimental system. 


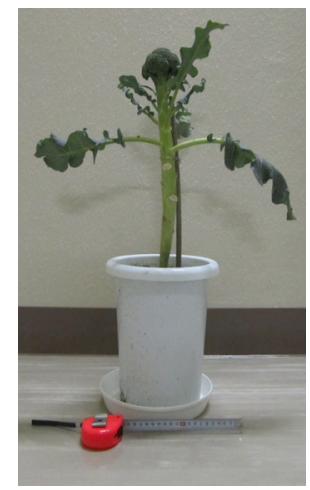

Figure 2: $\quad$ Photograph of the subjective plant (broccoli).

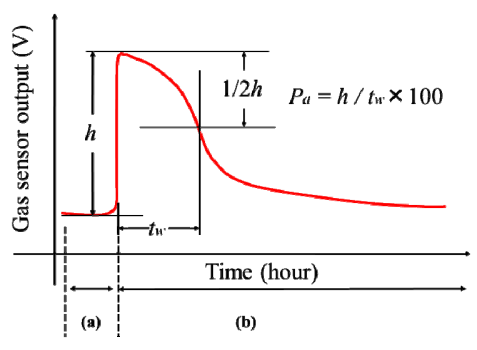

Figure 3: Purification capability of the subjective plant using the gas sensor characteristic.

\section{Experimental results}

It is obvious that the potential is changed according to environmental conditions and the growth situation but the detail is not still discovered. In this study, the research aim is to bring out the purification function of plants due to an irradiation light frequency, especially the nutrition intake and degradation of the nutrient source [16]. It is, however, very difficult and the goal is not made clear.

\subsection{Original characteristic and $v_{m 1}$}

As an example, the bioelectric potential and the gas sensor output are shown in Fig. 4. The potential is an original characteristic without pollutant for a minute under blue light $(2,177 l x)$, which is not the summation value $\left(v_{m 1}\right)$. Underneath is the gas sensor output, in which the experiment is carried out at 15:00 h and ethyl alcohol is injected using a micro syringe at 18:00 h. The concentration is about $8 \mathrm{ppm}$ in the chamber at that hour. The characteristic increases rapidly at the time of injection and decreases gradually, and it reaches its original state at an early hour by the purification capability of the broccoli. The h is about $0.4 \mathrm{~V}$ (corresponding to $8 \mathrm{ppm}$ ). $P_{a}$ is derived using the characteristic. The vegetable 
factory commonly accesses cheap electricity at night time and there is little disturbance to have an effect on the measurement at that time. The characteristics of temperature, humidity and atmospheric pressure which are measured at a time are shown in Fig. 5. Temperature is relatively constant at 20 degrees C. Humidity increases gradually from $60 \% \mathrm{RH}$ due to transpiration of the broccoli. The pressure changes slightly from 1007 to $1009 \mathrm{hPa}$.

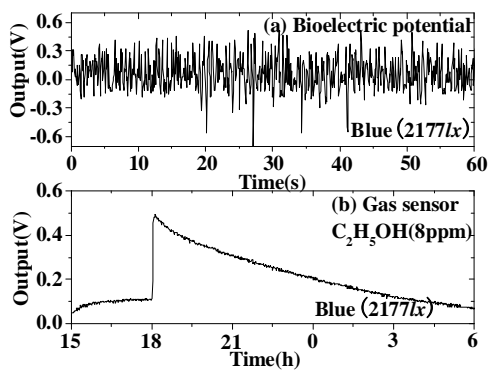

Figure 4: Original characteristic of the broccoli bioelectric potential (a) and the gas-sensor characteristics (b) to 8 ppm of ethyl alcohol as an air contaminant.

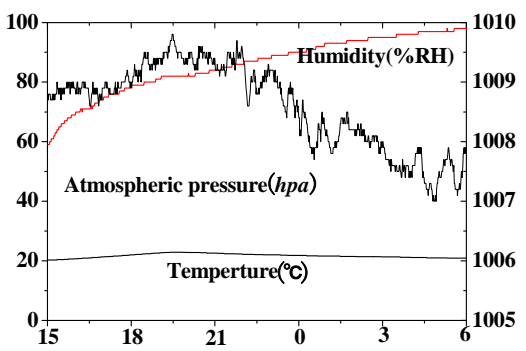

Figure 5: Characteristics of temperature, humidity and atmospheric pressure for 15:00 h to 18:00 $\mathrm{h}$.

It is difficult to derive the features from the original data of bioelectric potential shown in Fig. 4. For this reason, the summation value $\left(v_{m 1}\right)$ for a minute is newly adopted and the value is used to analyze the feature from the data. The value is obtained by adding the absolute data which are input every 0.1 seconds. The $v_{m 1}$ characteristic in the absence of the pollutant is adopted as a control in every irradiation light and the results are shown in Fig. 6. The characteristics in blue and white light are high but it is difficult to derive the features from the characteristics. For that reason, the $v_{m 1}$ is adopted. The $v_{m 1}$ histogram for the blue light is indicated in Fig .7. The average (ave, $v_{\text {m1-ave }}$ ) is $291.4[\mathrm{~V}]$ and the standard deviation $\sigma$ is 129.9. The coefficient of variation ( $\mathrm{cv}=\sigma / \mathrm{ave}$ ) is 0.446 . The average of $v_{m 1}\left(v_{m 1-a v e}\right)$ to each irradiation light is indicated in Fig. 8 as a bar 


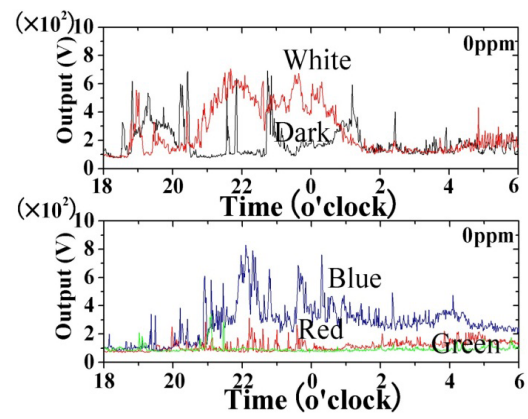

Figure 6: Summation value $\left(v_{m 1}\right)$ of bioelectric potential to each irradiation light frequency for one minute.

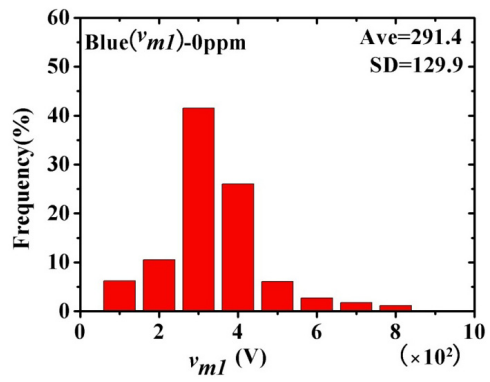

Figure 7: Histogram of $v_{m 1}$ under blue light. The average of $v_{m 1}\left(v_{m 1-a v e}\right)$ is 291.4 and the standard deviation is 129.9.

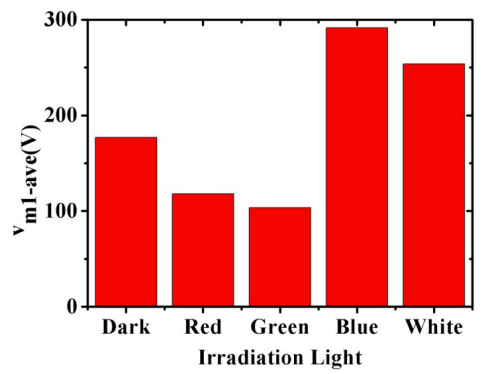

Figure 8: Relationship between irradiation lights and the average of $v_{m 1}\left(v_{m 1-a v e}\right)$.

graph. The value for blue light is high and the one for white light is second. The values make a little difference. It is thought that the characteristic shows some sort of the broccoli's physiological function for each light frequency. The relationship between the irradiation lights and the coefficient of variation (cv) of $v_{m 1}$ is shown in Fig. 9. The cv for red light is 0.288; the one for green is 0.495; 0.627 for white and the one in darkness is 0.564 . There is a tendency for the $\mathrm{cv}$ to increase as the light frequency increases. The ones in darkness (Dark) and under white light (White) in which there are various kinds of light frequencies 
are great. This tendency is the same as the result for cabbage [10]. It is thought that plants experience stress in darkness because they cannot photosynthesize. There have been no reports that the bioelectric potential shows a kind of plant stress. There is no definition of plant stress for now but the expression is used in literature [17].

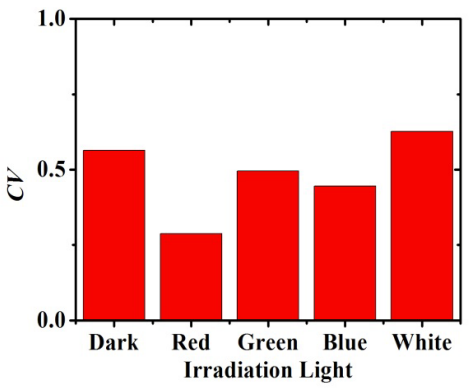

Figure 9: Relationship between irradiation lights and the coefficient of variation $(\mathrm{Cv})$ of $v_{m 1}$.

\subsection{Purification capability due to light frequency}

Plants' physiological function under light is not yet understood. The value of $v_{m 1}$ changes as a function of irradiating light frequency when there is no air pollutant like ethyl alcohol and the average $\left(v_{\text {m1-ave }}\right)$ also changes as described in the last section. The author has a feel for the changes which arise from plants' physiological function, and it also occurs by the presence or absence of an air pollutant. An efficient purifying system can be expected when the relationship between bioelectric potential and air pollutant under irradiation light is revealed and this enhances the use of the plant. Carbon dioxide is also removed effectively using the system. Ethyl alcohol is adopted as an air pollutant in this study and the relationship between the existence of the alcohol and $v_{m 1}$ is examined. The result can also contribute to the understanding of plant physiology. It is thought that plants absorb the pollutant (ethyl alcohol) as nutrition [14]. Photosynthesis plays a key role in the function. Actually, purification capability $\left(P_{a}\right)$ differs substantially in the cases of darkness and light irradiation [18]. Hydrogen molecules play a greater role in photosynthesis [19]. The molecule is produced by chemical decomposition of ethyl alcohol but there is an unclear point in the process.

The distribution characteristic of $v_{m 1}$ differs in the presence and the absence of a pollutant. In particular, there is a tendency for the coefficient of variance (cv) to increase. The experimental results of the relationship between the coefficient of variance and irradiation light are denoted in Fig. 10. In the figure, the five coefficients are indicated by bar graphs. Figure 10(a) shows the case for clean air and the (b) shows the one for injecting ethyl alcohol of $12 \mathrm{ppm}$. The coefficients for green and red lights increase remarkably in the presence of the alcohol. A large increase could not be recognized in other irradiation lights. It can be estimated that a peculiar phenomenon takes place under red and green lights. 


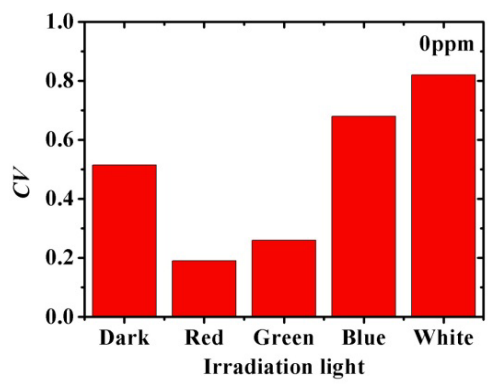

(a) $c v$ in clean air.

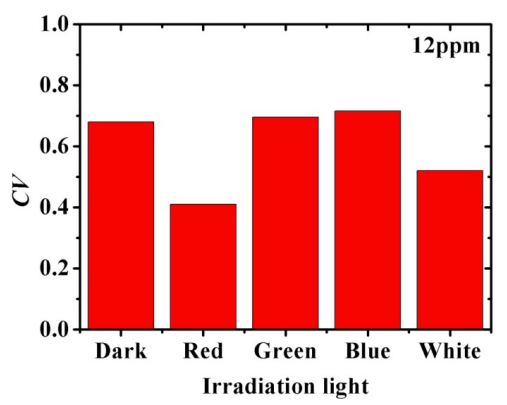

(b) $c v$ in $12 \mathrm{ppm}$ of gaseous ethyl alcohol.

Figure 10: Relationship between the coefficient of variance and irradiation light.

The relationship between the purification capability $\left(P_{a}\right)$ of broccoli and the average bioelectric potential $\left(v_{\text {m1-ave }}\right)$ in the presence of ethyl alcohol is shown in Fig. 11. It exhibits the result for 12 ppm as with Fig. 10(b). The plots for each irradiation light are mixed in the figure and the correlation coefficient is 0.65 . There is a correlation among the factors; the $P_{a}$ becomes greater as the $v_{\text {m1-ave }}$ becomes greater in general. The same trend exists in other vegetables [10]. Plants have a capability to return the polluting state to the clean state. Consequently, the $P_{a}$ becomes large with a highly polluting state. The relationship between $P_{a}$ and each concentration of ethyl alcohol under a blue light is shown in Fig. 12. Likewise, the one for red light is indicated in Fig. 13. The $P_{a}$ increases with increasing of the concentration of the pollutant and both characteristics have a tendency to be saturated. It is thought that there is a limitation to the $P_{a}$ over the concentration of $10 \mathrm{ppm}$, namely there is a physiology limitation to get the pollutant. Red and blue lights have a deep relationship with the photosynthetic function [20]. There is an Emerson effect in which the photosynthesis rate becomes larger when the plant is irradiated by mixed lights (for example red and blue) than monochromatic light. There is a report that the case of intensity ratio of 10:1 (red: blue light) is the greatest [4]. The understanding of plant growth can be provided if the bioelectric potential could be represented as a function of fertilizer consumption by the roots and amount of air-pollutant (including carbon 
dioxide) absorption by leaves, namely plant growth can be controlled in a plant factory. If the control could be understood, the accurate management of the production line in the vegetable factory is enabled and its corporatization of the factory moves ahead.

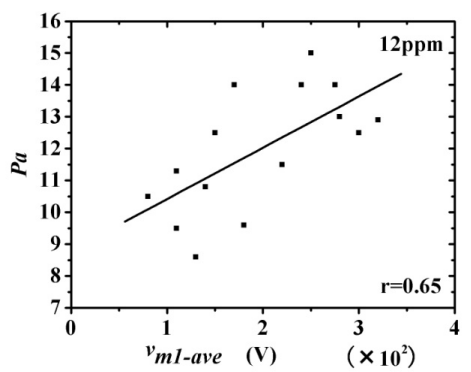

Figure 11: $\quad$ Relationship between $P_{a}$ and $v_{m 1-a v e}$.

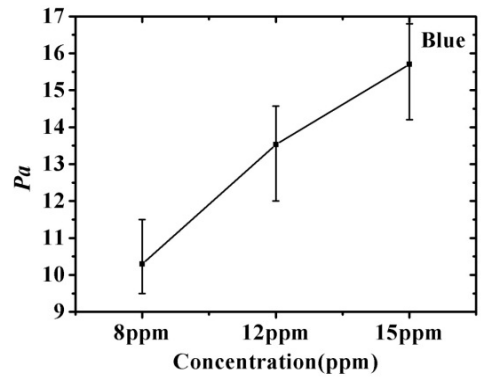

Figure 12: Relationship between $P_{a}$ and each concentration of ethyl alcohol under blue light.

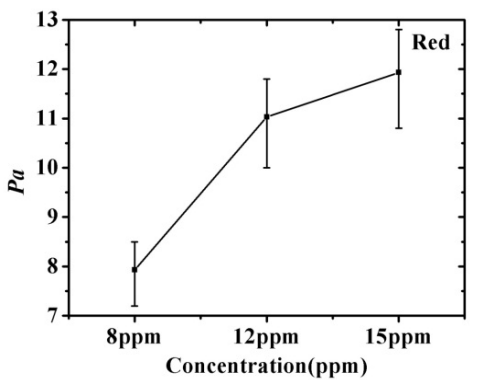

Figure 13: Relationship between $P_{a}$ and each concentration of ethyl alcohol under red light. 


\section{Conclusions}

The origins of plants go back much further than those of humans and is constantly evolving. Plants have various kinds of functions and the foremost important function is photosynthesis. There are also air-purification and transpiration for the function. It is necessary to examine the evolution process as well in order to clarify the plant-purification process. It is public knowledge that plant-purification capability varies by species [21]. The cause for the characteristics in the evolution process of plants should also be examined. That becomes a clue to protecting the global environment. The relationship between bioelectric potential and the irradiation light was examined in this study. The potential also changes due to environmental factors such as temperature, humidity, and atmospheric pressure [22]. It varies, depending on the presence or absence of an air pollutant and an irradiation light. It is difficult to derive the features directly from the original characteristic of the potential because it varies momentarily. Therefore, the value which is summed up from the input signals of the potential for a minute is newly adopted and ethyl alcohol is adopted as an air pollutant. Carbon dioxide should be adopted essentially. An effective measure for maintaining the global environment will be derived if the highly-efficient purification function of plants could be introduced using the bioelectric potential. Broccoli is used as a subjective plant, which is a kind of ordinal vegetable. As for the results, it becomes clear that the potential becomes great and the purification capability also becomes great due to the irradiation light. As a particular attention, removing the capability of the plant for the pollutant becomes high as the frequency of the irradiation light increases. There is a positive correlation between the summed potential and the capability. It is thought that this result will be applied to vegetable production and lead to production management.

The rough relationship between the bioelectric potential and light frequency was investigated in this study. It does not become obvious which physiological activity of plant causes the relationship. It is necessary to investigate the purification capability as a matrix in association of plant species and chemicals under the mixed light frequencies. And it is necessary to bring out the derivation of the capability from the evolution process of plants.

\section{References}

[1] Yuzuru Fushimi: "Physics for beginning of life and evolution”, Kyoritsusyuppan (2002) (in Japanese).

[2] Ayako Sawada and Takashi Oyabu: "Healing Effects of Foliage Plants Using Physiological and Psychological Characteristics", Sensors and Materials, Vol. 22, No. 7, pp. 387-396 (2010).

[3] Masamoto Takatsuji: "Illustrated guide for plant factory", Nikkan Kogyo Shinbunsha (2010) (in Japanese).

[4] Masamoto Takatsuji: “Totally controlled plant factory”, Ohmsha (2010) (in Japanese). 
[5] Toyoki Kozai: "Plant factory using sunlight”, Ohmsha (2009) (in Japanese).

[6] Hideo Ikeda: "Plant factory and the business", Nihon Keizai Shinbunsha (2010) (in Japanese).

[7] Masamoto Takatsuji and Yasuhiro Mori: "Plant factory using LED”, Nikkan Kogyo Shinbunsha (2011) (in Japanese).

[8] Ryukichi Kijima, Yuta Kato: "The Charge of Food and Health by Plant Factory”, The Journal of The Electrical Engineers of Japan, Vol. 131, No. 11, pp. 764-767 (2011) (in Japanese).

[9] Noboru Noguchi: "Innovation of Agricultural Technology using ICT”, Journal of The Society of Instrument and Control Engineering, Vol. 50, No. 12, pp. 1025-1075 (2011) (in Japanese).

[10] Masaki Tokuda, Lixin Shao, Takashi Oyabu, Hidehito Nanto: "Plant bioelectric potential of hard-leaf cabbage to irradiation-light frequency", The Institute of Electrical Engineers of Japan, Vol. 132, No. 6, Scene (2012.6) (in Japanese).

[11] Haruo Sugi: "What is a bioelectric signal?”, Kodansya (2006) (in Japanese).

[12] Frantisek Baluska, Srefano Mancuso, Dieter Volkmann: “Communication in Plants”, Springer (2006).

[13] Kang Baosheng, Shin-ichi Shibata, Ayako Sawada, Takashi Oyabu and Haruhiko Kimura: “Air Purification Capability of Potted Phoenix Roebelenii and Its Installation Effect in Indoor Space”, Sensors and Materials, Vol. 21, No. 8, pp. 445-455 (2009).

[14] Henry H. Tabak, Stephen A. Quave, Charles I. Mashini and Edwin F. Barth: "Biodegradability studies with organic priority pollutant compounds”, Journal WPCF, Vol. 53, No. 10, pp. 1503-1518 (1981).

[15] Takashi Oyabu, Takeshi Onodera, Haruhiko Kimura, and Yoshihiko Sadaoka:’Purification Ability of Interior Plant for Removing of Indoor-Air Polluting Chemicals Using a Tin Oxide Gas Sensor”, Journal of Japan Society for Atmospheric Environment, Vol. 34, No. 6, pp. 319-325 (2001).

[16] Ki Ando, Yuki Hasegawa, Tamaki Yaji, Hidekazu Uchida: "Study of Plant Bioelectric Potential Response due to Photosynthesis Reaction”, The Institute of Electrical Engineers of Japan, Sec. E, Vol. 131, No. 9, pp. 337342 (2011) (in Japanese).

[17] A.H. Fitter, R.K.M. Hay: "Environmental Physiology of Plants”, Academic Press (1981).

[18] Masaki Fujii, Masahiro Tani, Ayako Sawada, Kouji Ueshima, Kousaku Nogami and Takashi Oyabu: "Purification characteristic of pothos for airborne contaminants according to growth conditions", The society of Environmental Instrumentation, Control and Automation, Vol. 12, No. 1, pp. 29-34 (2007) (in Japanese).

[19] Yoshio Masuda: "Plant physiology”, Baifukan (1999) (in Japanese).

[20] Masamoto Takatuji: "Basic and Practice for Plant Factory", Shokabo (2007) (in Japanese). 
[21] Sei-ichiro Kamisaka, Kazuhiko Nishitani, Naoki Sakurai, Ei-ichi Tanimoto, Jyun-ichi Ueda, Shinobu Watanabe: "Introduction to Plan Biological Science”, Baifukan (2000) (in Japanese).

[22] Tatsuya Shimbo, Takashi Oyabu, Yuki Hasegawa, Shigeki Hirobayashi and Kozaburo Takenaka: "Bioelectric Potential Characteristic of Potted Plant for Temperature Change in a Residential Space”, The Institute of Electrical Engineers of Japan, Sec. E, Vol. 125, No. 6, pp. 253-258 (2005) (in Japanese). 